\title{
Atypical Presentation of Isoniazid induced Left Sided Pleural Effusion in Case of Right Sided Koch's on Anti-Tubercular Drugs: Case Report
}

\author{
Dr. BharatveerManchanda(Assist. Prof. $)^{1}$, Dr. Shirobhi Sharma(JR-III $)^{2}$, \\ Dr. SaloniMehra(JR-III $)^{3}$, Dr. NishantTayal(JR-III $)^{4}$, Dr. UmeshVerma(JR-I ${ }^{5}$. \\ Subharti Medical College, Meerut
}

\section{Introduction}

The clinical scenario of a new or worsening pleural effusion following the initiation of anti-tubercular therapy has been classically referred to as a 'paradoxical' pleural response, presumably explained by an immunological rebound phenomenon. Emerging evidence suggests that there also may be a role for a lupusrelated reaction in the pathophysiology of this disorder.

\section{Case Report}

A 40 years male presented with history of 2 month dyspnoea, cough with expectoration and palpitations. His vitals were stable except for respiratory rate : $28 / \mathrm{min}$; saturation was $90 \%$. On examination, bronchial breathing was present over right upper lobe with diffuse rhonchi all over the lung fields. Sputum was AFB positive. Radiological investigation showed cavity in right upper zone. Patient was diagnosed as a case of pulmonary tuberculosis and ATT was started. After 3 weeks of treatment, patient dyspnea increased.

Chest X-ray was done which showed left sided pleural effusion. Pleurocentesis was performed and showed:colour was orange tinged. Gram and AFB (acid-fast bacilli) staining of the pleural fluid were negative. The $\mathrm{pH}$ of the fluid was 7.2; WBC count, 80 cells/mm3 [69\% polymorphonuclear leukocytes (PMNs), 23\% monocytes]; total fluid protein, $4.3 \mathrm{~g} / \mathrm{dL}$; LDH (lactate dehydrogenase), $1250 \mathrm{IU} / \mathrm{L}$; and glucose, $10 \mathrm{mg} / \mathrm{dL}$. At the same time, the total serum protein was $6.9 \mathrm{~g} / \mathrm{dL}$; serum LDH was $566 \mathrm{IU} / \mathrm{L}$.ADA (adenosine deaminase) level was 20(normal) and ANA (antinuclear antibodies) of the fluid came out to be positive.INH was discontinued and rifampin was started for latent tuberculosis treatment. Over the next few days, his dyspnea improved, his cough resolved. On hospital day 20, further testing revealed positive double-stranded DNA (dsDNA) antibodies he was started on oral prednisone for drug-induced lupus, likely secondary to INH therapy.After 25 total days of hospitalization, the patient was discharged on oral prednisone (as well as his other medications). A repeat chest radiograph done 2 weeks later showed complete resolution of the pleural effusion

\section{Discussion}

- A wide range of medications have the potential to induce side effects that mimic autoimmune syndromes.

- INH has been implicated as one of over 80 medications implicated in drug-induced lupus erythematosus (DILE), which has been defined as a lupus-like syndrome that is temporarily related to continuous drug exposure (from 1 month to over a decade) and resolves after cessation of the offending pharmacologic agent.

- $\quad$ INH is known to cause a positive ANA in $25 \%$ of patients and clinically apparent drug-induced lupus in approximately $1 \%$ of patients. The underlying pathophysiology has not yet been elucidated.INH-induced pleural effusion usually begins 2 to 12 weeks after starting the medication and regresses after a change of therapy or introduction of steroids.

- In our patient pleural effusion began after approximately 3 weeks after the start of INH . While the exact pathogenic mechanism of INH-induced pleural effusion remains unclear, this indicates that the disease entity may have an underlying inflammatory component.

- The dramatic improvement of his autoimmune symptoms after starting prednisone, an anti-inflammatory agent and immunosuppressant, partially supports this notion.

- In most cases of described DILE, the presence of anti-dsDNA. It is also uncommon to have symptomatic pleural effusions due to a medication. As well, to the best of our knowledge, there has been only few reported case of drug-induced lupus with positive anti-dsDNA antibodies.

- Postulates have been introduced to explain the phenomenon of INH-induced pleural effusion, including "immunological rebound"7 or an effect between mycobacterial products and improving host immunity. 


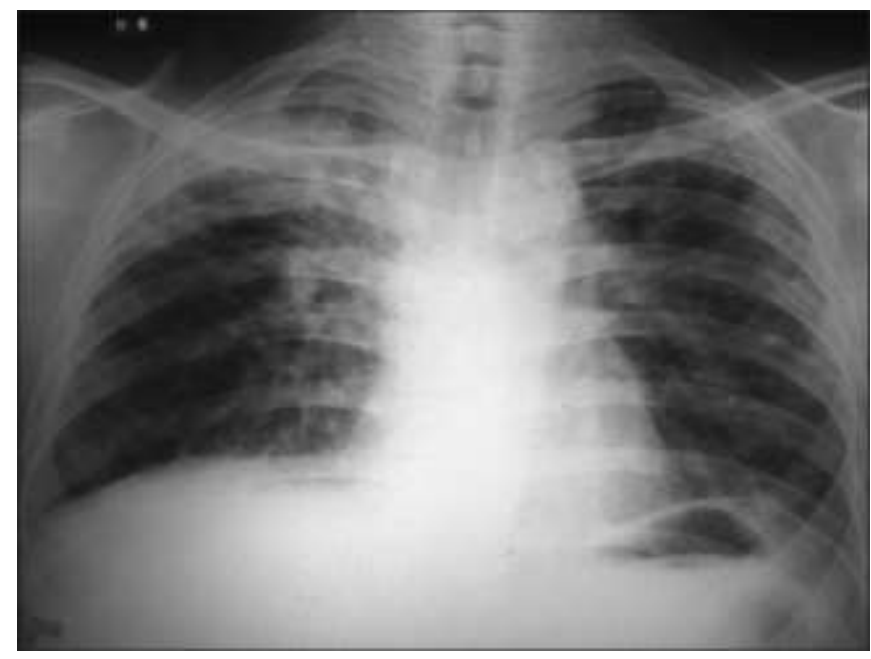

series of $\mathrm{x}$-ray showing regression of effusion

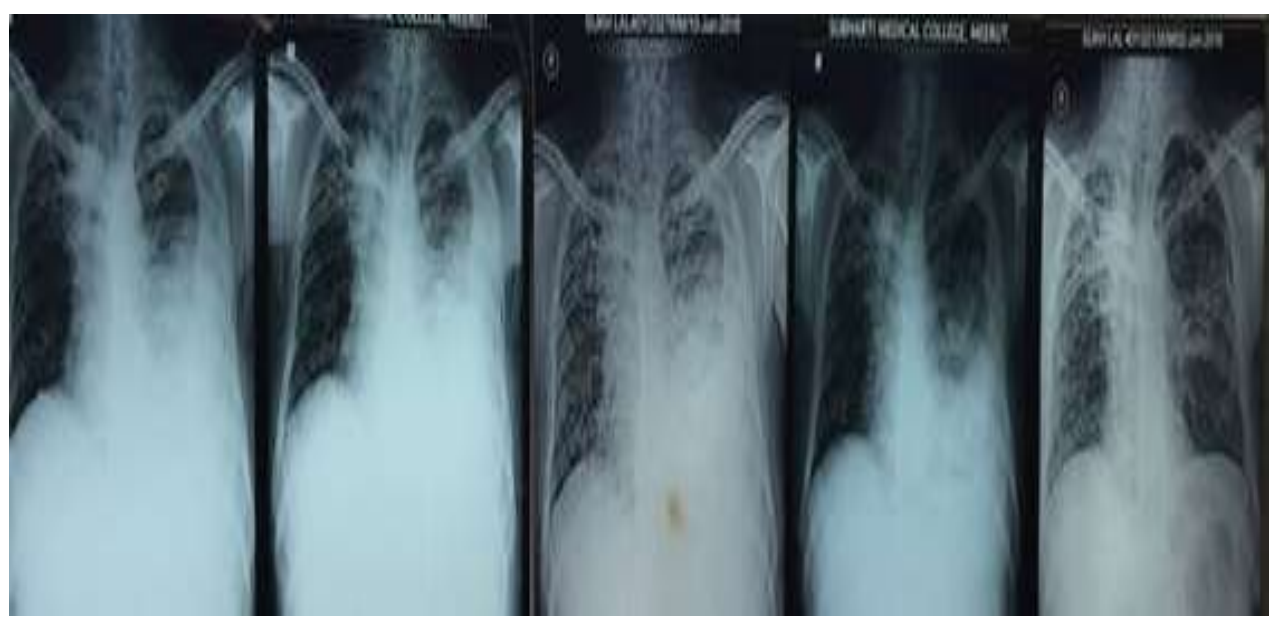

